\title{
CALIDAD DE ATENCION DEL PARTO SEGÚN PUERPERAS DEL SERVICIO DE OBSTETRICIA DE UN HOSPITAL GENERAL DE PERÚ
}

\section{QUALITY OF DELIVERY CARE ACCORDING TO PUERPERAS OF THE OBSTETRICS SERVICE OF A GENERAL HOSPITAL OF PERU}

Jean Aquije-Munañte ${ }^{1, a}$, Ana Kuroki-De-Kawata ${ }^{1, a, b}$.

1. Facultad de Medicina Humana. Universidad Nacional San Luis Gonzaga. Ica, Perú.

a. Médico cirujano.

b. Ginecóloga obstetra.

Correspondencia:

Aquije Muñante Jean Pierre Dirección: Av. 28 de Julio \#110-Subtanjalla

Correo:

aquije_jam94@outlook.com Celular: 956554464

\section{Contribuciones De Autoría:}

JAM ha participado en la concepción y diseño del artículo, recolección de los datos. AKI participó en el análisis estadístico de datos. Los autores participaron en la interpretación de los datos, redacción del manuscrito, revisión crítica del manuscrito y aprobaron la versión final.

Conflicto De Intereses: Los autores declaran no tener conflictos de interés. Financiamiento: Autofinanciado.

\section{Como Citar}

Aquije- Muñante J. Kuroki- De Kawata A. Calidad de atención del parto según puerperas del servicio de obstetricia de un Hospital General de Perú. Rev méd panacea. 2020; 9(1): 13-22. doi: https://doi.org/10.35563/rmp.v9i1.291

Recibido: 09 - 01 - 2020

Aceptado: 02 - $02-2020$

Publicado: 06 - 03 - 2020

\section{RESUMEN}

Objetivo: Determinar la calidad de atención del parto según puérperas del Servicio de obstetricia del Hospital Regional de Ica, Mayo -Setiembre 2019. Materiales y métodos: Estudio descriptivo, observacional, prospectivo y transversal, muestra de estudio conformada por 211 puérperas, aplicando como técnicas la entrevista y encuesta, como instrumento de recolección de datos el cuestionario SERVPERF de calidad de atención, medido en una escala de 1 a 6 según satisfacción percibida, validado internacionalmente y ampliamente utilizado en la atención del parto. Resultados: La calidad de atención del parto, según satisfacción de las puérperas es en promedio de nivel muy bueno $X \otimes=5.16$, evidenciando que $70.1 \%$ percibe la calidad de atención de nivel muy bueno (70.1\%), nivel bueno $26.1 \%$, nivel malo $3.3 \%$ y nivel extremadamente bueno $0.5 \%$. Por dimensiones la calidad de atención del parto es de nivel muy bueno para cada una de ellas, siendo las dimensiones mejores valoradas los elementos tangibles $X \nabla=5.43$, la empatía $X \nabla=5.32$ y la confianza $X \otimes=5.18$; y menos valoradas la dimensión respuesta rápida $X \nabla=5.05$ y seguridad $X \nabla$ $=5.04$. Se encontró asociación estadística significativa entre las variables sociodemográficas: edad ( $p$ 0.013), grado de instrucción ( $p$ 0.000), y estado civil ( $p$ 0.024). Las variables no asociadas fueron la ocupación ( $p$ 0.197), procedencia ( $p$ 0.475), y personal que atendió el parto ( $p$ 0.836). Conclusiones: La calidad de atención del parto, según satisfacción de las puérperas atendidas es de nivel muy bueno, existiendo un 3.3\% de insatisfacción o nivel malo de calidad.

Palabras clave: Calidad de la Atención de Salud, Parto Obstétrico, Periodo Posparto, Servicio de ginecología y obstetricia en hospital (Fuente: DeCS BIREME).

\section{ABSTRACT}

Objective: Determine the quality of childbirth care according to postpartum women of the Obstetrics Service of the Regional Hospital of Ica, May-September 2019. Materials and methods: Descriptive, observational, prospective and cross-sectional study, study sample formed by 211 postpartum, using the interview and survey techniques as techniques, as a tool for data collection, the SERVPERF quality of care questionnaire, measured on a scale of 1 to 6 according to perceived satisfaction, internationally validated and widely used in childbirth care. Results: The quality of delivery care, according to the satisfaction of the postpartum women, is on average very good level $X \mathrm{X}=5.16$, showing that $70.1 \%$ perceive the quality of care at a very good level $(70.1 \%)$, good level $26.1 \%$, level Bad $3.3 \%$ and extremely good level $0.5 \%$. By dimensions, the quality of childbirth care is of a very good level for each of them, the tangible elements being tangibles $=X \nabla=5.43$, the empathy $X \nabla=5.32$ and the confidence $X \otimes=5.18$; and the value of the rapid response dimension $X \nabla=5.05$ and safety $X \nabla=5.04$ are less valued. A significant statistical association was found between the sociodemographic variables: age ( $p 0.013)$, degree of instruction ( $p 0.000)$, and marital status ( $p$ 0.024). Non-associated variables were occupation ( $p 0.197)$, origin ( $p 0.475)$, and personnel who attended the delivery ( $p$ 0.836). Conclusions: The quality of childbirth care, according to satisfaction of the treated women is of a very good level, with $3.3 \%$ of dissatisfaction or poor level of quality.

Keywords: Quality of Health Care, Delivery, Obstetric, Postpartum Period, Obstetrics and Gynecology Department, Hospital (Source: DeCS BIREME). 


\section{INTRODUCCIÓN}

Se ha considerado como indicador de calidad para medir el cuidado en salud a la satisfacción del paciente. Considerando la mortalidad materna como un indicador negativo de salud, en el 2015 se estimaron unos 303000 muertes de mujeres durante el embarazo y el parto o después de este a nivel mundial (1).

La salud de la mujer es un derecho fundamental (2), y la atención del parto debe realizarse basándose en evidencia científica pero siempre desde una perspectiva humana y centrado en la persona (Parto humanizado) (3); comprendiendo como Atención Humanizada del Parto (AHP) como la observación de una serie de atributos con el fin de brindarle a la gestante el cuidado y permitirle una vivencia satisfactoria del trabajo de parto y el parto, y garantizando a su vez esta vivencia a la población de gestantes de bajo nivel socioeconómico, de alto riesgo, y sobre todo adolescentes, que son la población más vulnerable.

El parto es un proceso fisiológico de gran dolor según la perspectiva de cada mujer (4) y momento de gran vulnerabilidad, por lo que requiere de un proceso de cuidado continuo. Sin embargo, con el avance en la ciencia y las tecnologías sanitarias, se han impulsado el desarrollo de un enfoque de medicalización y tecnificación del proceso del parto (5). Produciendo un abuso de episiotomías y aumento de la tasa de cesáreas mayor al 20\%, la cual la OMS no justifica $(6,7)$. Además se ha evidenciado en estudios como el de Rodríguez la relación de un menor grado de satisfacción en las usuarias con una experiencia anterior por cesárea (8).

Se vienen utilizando estrategias dirigidas a mejorar la calidad de atención de los servicios de salud materno, como en el año 2016 con el "parto humanizado en Perú: parto vertical" (9). Se puede concluir en los diferentes trabajos de investigación que una práctica no violenta en la atención del parto aseguraría el respeto de los derechos fundamentales de la mujer y del recién nacido, reduciendo así las complicaciones perinatales y la mortalidad materno-neonatal. En este contexto se podría implementar una capacitación de comunicación para médicos, y con este buscar el empoderamiento de la madre (10).

Aunado a lo anterior y debido a la creciente instrumentalización del parto ha dificultado que se preste la suficiente atención a la mujer durante este proceso, lo cual puede influir en su adaptación a la maternidad y en su bienestar físico y emocional, al vínculo madre-hijo y por ende a la satisfacción de la madre con la atención recibida. En el presente estudio se buscó determinar la calidad de la atención según satisfacción de las puérperas del Servicio de Obstetricia del Hospital regional de Ica; con el fin de contribuir en reducir la mortalidad materna y mejorar y brindar una atención de calidad integral impartido desde el interno de medicina hasta el médico especialista y todo el personal involucrado.

\section{MATERIALES Y MÉTODOS}

Se realizó un estudio observacional, prospectivo, transversal y descriptivo. La población objetivo estuvo constituida por todas las pacientes puérperas inmediatas atendidas en el Servicio de obstetricia del Hospital Regional de Ica en los meses de Mayo a Setiembre del 2019, la cual fue en total 463. La muestra fue obtenida mediante Muestreo probabilístico, y selección de adolescentes mediante el muestreo aleatorio simple (selección al azar), obteniendo así una muestra de 211 puérperas inmediatas. Los criterios de inclusión para esta investigación fueron: Puérperas que acepten participar y firmen el consentimiento informado (en el caso de ser menor de edad junto con la firma y consentimiento del padre o apoderado), mayores de 15 años de edad, puérperas inmediatas con recién nacido vivo y sin complicaciones postparto. Los criterios de exclusión fueron Madre con: alteraciones del habla o comprensión, no fueron atendidas en el Hospital Regional de Ica.

La recolección de datos se realizó mediante la entrevista cara a cara, en donde se les orientó a las puérperas participantes sobre el estudio y su objetivo, la cual aceptara o no entrar al estudio, firmando el consentimiento informado y en el caso de ser menor de edad se pedirá el consentimiento de su padre o apoderado. La duración aproximada de esta entrevista fue de 20 minutos. El entrevistador verificó que todas las preguntas estén respondidas; sin embargo, al finalizar la aplicación de los instrumentos de la investigación (ficha de recolección de datos y la Escala SERVPERF, el cual fue evaluado por un grupo de expertos en el área de calidad y opinaron que es aplicable a la población en estudio) realizó el control de calidad previo a la digitación a fin de identificar posibles errores o vacíos.

El procesamiento y análisis se trabajó con un nivel de significancia estadística del 5\%. Para el procesamiento de los datos se utilizó el software Microsoft Excel 2016 y SPSS versión 25.0. Seguidamente se procedió al análisis estadístico con distribución de frecuencias, medidas de tendencia central y de dispersión, para las variables categóricas se presentan como proporciones. La asociación de variables se realizó mediante prueba de chi cuadrado. Con todo esto se procedió a realizar tablas de frecuencias, porcentajes y figuras estadísticas, donde se ordenaron de lo más general al más específico. 


\section{RESULTADOS}

Tabla1. Características sociodemográficas, en puérperas del Servicio de Obstetricia del Hospital Regional de Ica, Mayo - Setiembre 2019

\begin{tabular}{|c|c|c|}
\hline Variable & $\mathbf{N}=\mathbf{2 1 1}$ & $100 \%$ \\
\hline Edad & $\mathbf{n}$ & $\%$ \\
\hline 15 a 19 años & 31 & 14.7 \\
\hline 20-25 años & 66 & 31.3 \\
\hline 26-30 años & 52 & 24.6 \\
\hline $31-40$ años & 57 & 27.0 \\
\hline 41-43 años & 5 & 2.4 \\
\hline Grado de instrucción & $\mathrm{n}$ & $\%$ \\
\hline Primaria incompleta & 1 & 0.5 \\
\hline Primaria completa & 7 & 3.3 \\
\hline Secundaria incompleta & 49 & 23.2 \\
\hline Secundaria completa & 92 & 43.6 \\
\hline Superior No universitaria & 43 & 20.4 \\
\hline Superior Universitaria & 19 & 9.0 \\
\hline \multicolumn{3}{|l|}{ Estado civil } \\
\hline Soltera & 30 & 14.2 \\
\hline Casada & 29 & 13.7 \\
\hline Conviviente & 152 & 72.0 \\
\hline \multicolumn{3}{|l|}{ Ocupación } \\
\hline Ama de casa & 181 & 85.8 \\
\hline Independiente & 4 & 1.9 \\
\hline Dependiente & 26 & 12.3 \\
\hline \multicolumn{3}{|l|}{ Procedencia } \\
\hline Urbano & 170 & 80.6 \\
\hline Rural & 41 & 19.4 \\
\hline
\end{tabular}

Según características sociodemográficas y de atención del parto, se observa que la mayor proporción de puérperas encuestadas tienen: una edad entre 20 a 25 años (en 66 casos, 31.3\%), una instrucción educativa secundaria completa (en 92 casos, 43.6\%), como estado civil convivientes (en 152 casos, 72\%), tienen como ocupación ama de casa (en 181 casos, 85.8\%), y con mayor frecuencia las puérperas provienen de zonas urbanas (en 170 casos, 80.6\%).
Gráfico 1. Atención del parto, según personal de salud, en puérperas del Servicio de Obstetricia del Hospital Regional de Ica, Mayo - Setiembre 2019

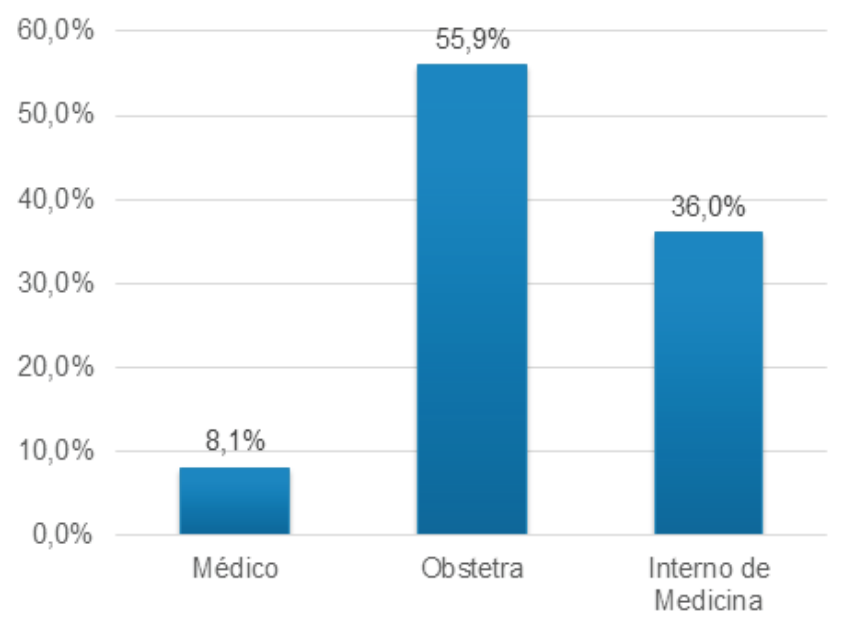

Según atención de parto, se observa que el procedimiento fue realizado mayormente por el profesional obstetra con 55.9\%.

Tabla2. Calidad de atención del parto según puérperas del Servicio de Obstetricia del Hospital Regional de Ica, Mayo - Setiembre 2019: Análisis Global

\begin{tabular}{lccc}
$\begin{array}{lccc}\text { Calidad de Atención del } \\
\quad\end{array}$ Parto: Global & $\mathrm{N}^{\circ}$ & $\%$ & Promedio \\
Malo & 7 & 3.3 & \\
Bueno & 55 & 26.1 & $\bar{X}=5.16$ \\
Muy bueno & 148 & 70.1 & $D S=0.56$ \\
Extremadamente bueno & 1 & 0.5 & \\
$\quad$ Total & 211 & $\mathbf{1 0 0 \%}$ & \\
\hline
\end{tabular}

Se observa que la calidad de atención del parto es percibida de manera global como buena. La puntuación que más se repitió fue 5 (muy bueno). El promedio aritmético sitúa a la calidad de atención global en el nivel bueno ( $X \nabla=5.16$ ). Asimismo, se desvían de 5.16, en promedio, 0.56 unidades de la escala. Ninguna persona califico de manera global a la calidad de la atención de manera muy desfavorable (no hay "1 o 2 "). Las puntuaciones tienden a ubicarse en valores medios elevados. 
Tabla3. Análisis de la Calidad de atención del parto por ítems, según puérperas del Servicio de Obstetricia del Hospital Regional de Ica, Mayo - Setiembre 2019

\begin{tabular}{|c|c|c|c|c|c|c|c|c|}
\hline \multirow[b]{2}{*}{$\mathrm{N}^{\circ}$} & \multirow[b]{2}{*}{ ítems } & \multicolumn{6}{|c|}{ Total $100 \%$} & $\bar{X}$ \\
\hline & & 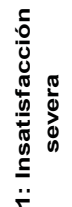 & 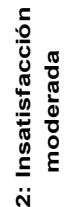 & 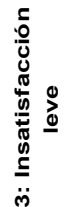 & 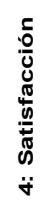 & 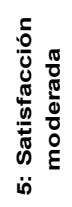 & 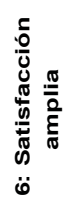 & 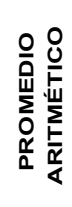 \\
\hline
\end{tabular}

Dimensión: Respuesta rápida

1 ¿Fue usted atendida inmediatamente a su llegada al hospital?

$3.8 \%$

$3.3 \%$

$4.3 \% \quad 19.0 \%$

$22.3 \%$

$47.4 \%$

4.94

¿En momentos en los cuales necesito

2 la ayuda/atención del personal, la atendieron rápida y oportunamente?

$\begin{array}{lllllll}1.9 \% & 0.0 \% & 10.0 \% & 13.7 \% & 27.5 \% & 46.9 \% & 5.05\end{array}$

3 ¿Fue trasladada rápidamente a centro obstétrico?

$\begin{array}{lllllll}0.0 \% & 3.3 \% & 5.2 \% & 15.6 \% & 26.1 \% & 49.8 \% & 5.13\end{array}$

\section{Dimensión: Empatía}

¿El personal de centro obstétrico la

4 trato con amabilidad, respeto $y$ paciencia?

5 ¿Recibió algún grito o maltrato por el personal de salud?

$0.5 \%$

$2.4 \%$

$14.2 \%$

$14.2 \%$

$65.9 \%$

5.34

6 ¿Fue el personal de salud indiferente al dolor que usted sentía?

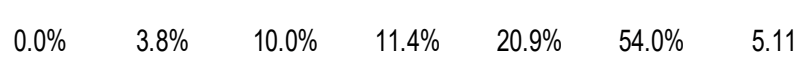

\section{Dimensión: Seguridad}

¿Su atención estuvo a cargo del

7 médico, obstetra o interno de medicina con medico?

8 ¿Se le dejo sola durante largos periodos de tiempo?

9 ¿El personal de salud mostró interés genuino en su bienestar?

¿El personal de centro obstétrico le 10 mostró interés para solucionar cualquier problema que se presentó durante su $0.0 \%$ $2.8 \%$ $0.0 \%$ parto?

11 ¿Se le pidió permiso antes de realizar cualquier procedimiento? 
12 ¿Pudo acompañarla su esposo 0

$\begin{array}{lllllll}\text { familiar durante el momento del parto? } & 20.4 \% & 3.8 \% & 21.3 \% & 20.9 \% & 17.5 \% & 16.1 \%\end{array}$

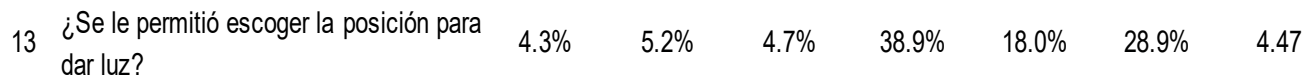

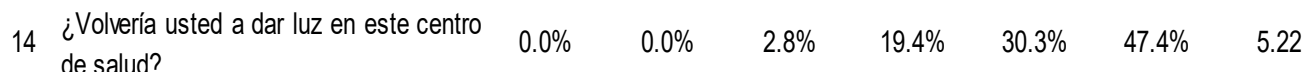

\begin{tabular}{|c|c|c|c|c|c|c|c|c|}
\hline \multirow[b]{2}{*}{$\mathrm{N}^{\circ}$} & \multirow[b]{2}{*}{ ítems } & \multicolumn{6}{|c|}{ Total $100 \%$} & \multirow{2}{*}{ 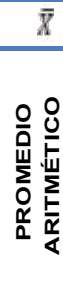 } \\
\hline & & 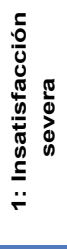 & 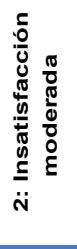 & 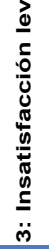 & 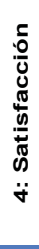 & 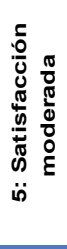 & 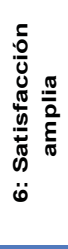 & \\
\hline
\end{tabular}

Dimensión: Confianza

¿El médico u obstetra que la atendió mantuvo suficiente comunicación con

15 usted o sus familiares para explicarles su situación en cada momento del parto?

16 ¿Pudo tomar o comer algo durante el trabajo de parto?

$\begin{array}{lllllll}0.0 \% & 0.0 \% & 0.0 \% & 14.2 \% & 29.9 \% & 55.9 \% & 5.41\end{array}$

17 ¿Se le permitió acariciar a su bebé apenas nació?

18 ¿Se le brindó la adecuada privacidad durante los exámenes realizados?

19 ¿Recomendaría a un familiar o amigo a que den a luz en este establecimiento?

$\begin{array}{lllllll}0.0 \% & 0.0 \% & 1.9 \% & 13.7 \% & 26.1 \% & 58.3 \% & 5.40\end{array}$

\section{Dimensión: Elementos Tangibles}

20 ¿Los ambientes de centro obstétrico estuvieron limpios y cómodos?

$\begin{array}{llllll}0.0 \% & 0.0 \% & 2.8 \% & 14.2 \% & 21.3 \% & 61.6 \%\end{array}$

¿Se sentía confortable en el ambiente que se encontraba durante el trabajo de $\quad 0.0 \% \quad 0.0 \% \quad 3.3 \% \quad 14.7 \% \quad 22.3 \% \quad 59.7 \% \quad 5.38$ parto?

¿El personal de salud contó con

22 equipos disponibles y materiales necesarios para su atención? 
Se observa que en determinados ítems de la calidad de atención del parto existe un cierto porcentaje de puérperas con insatisfacción: En la dimensión respuesta rápida la mayor insatisfacción se debe al no tener una rápida ayuda/atención del personal de salud en 11,9\%. En la dimensión empatía la mayor insatisfacción se debe al percibir indiferencia del personal para tratar su dolor en 13,8\%. En la dimensión seguridad la mayor insatisfacción se debe al no permitírsele estar acompañada con su pareja o familiar en $45,5 \%$. En la dimensión confianza la mayor insatisfacción se debe al no permitírsele consumir alimentos durante el trabajo de parto en $19,9 \%$. Siendo la dimensión elementos tangibles la mejor valorada y con menor porcentaje de insatisfacción. En general la puntuación de cada ítem tiende a ubicarse en el nivel medio alto.

Gráfico 2. Dimensiones de la calidad de atención del parto según promedios ponderados, según puérperas del Servicio de Obstetricia del Hospital Regional de Ica, Mayo - Setiembre 2019

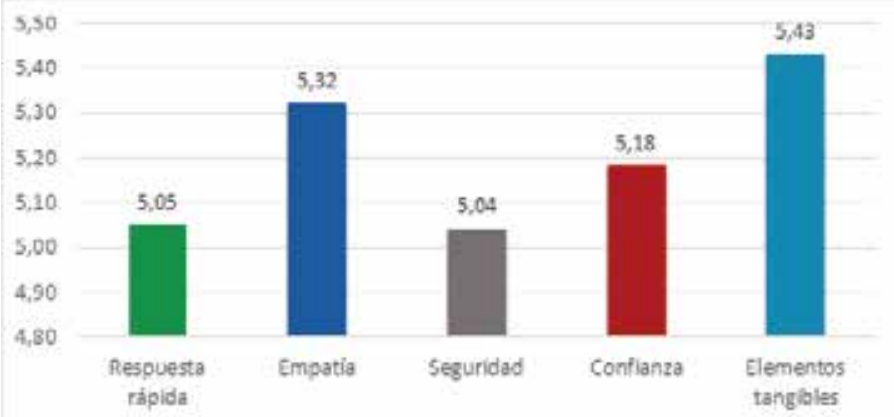

Se observa que en todas las dimensiones de la calidad de atención del parto fueron valoradas por las puérperas de nivel muy bueno. La dimensión con mayor nivel de satisfacción es los Elementos tangibles. Las dimensione menos valoradas fueron Respuesta rápida y Seguridad.

Tabla4. Edad y calidad de atención del parto en puérperas del Servicio de Obstetricia del Hospital Regional de Ica, Mayo - Setiembre 2019

\begin{tabular}{|c|c|c|c|c|c|c|c|c|c|c|c|}
\hline \multicolumn{12}{|c|}{ Calidad de atención } \\
\hline \multirow[t]{2}{*}{ Edad } & \multicolumn{2}{|c|}{ Malo } & \multicolumn{2}{|c|}{ Bueno } & \multicolumn{2}{|c|}{ Muy bueno } & \multicolumn{2}{|c|}{ Extremad. bueno } & \multicolumn{3}{|c|}{ Prueba estadística } \\
\hline & $\mathbf{N}^{\circ}$ & $\%$ & $\mathbf{N}^{\circ}$ & $\%$ & $\mathbf{N}^{0}$ & $\%$ & $\mathbf{N}^{\circ}$ & $\%$ & $\mathrm{x}_{2}$ & gl & p \\
\hline 15 a 19 años & 1 & 0.5 & 0 & 0.0 & 30 & 14.2 & 0 & 0.0 & \multirow{6}{*}{25.31} & \multirow{6}{*}{12} & \multirow{6}{*}{0.013} \\
\hline 20-25 años & 2 & 0.9 & 15 & 7.1 & 48 & 22.7 & 1 & 0.5 & & & \\
\hline 26-30 años & 4 & 1.9 & 16 & 7.6 & 32 & 15.2 & 0 & 0.0 & & & \\
\hline 31-40 años & 0 & 0.0 & 23 & 10.9 & 34 & 16.1 & 0 & 0.0 & & & \\
\hline 41-43 años & 0 & 0.0 & 1 & 0.5 & 4 & 1.9 & 0 & 0.0 & & & \\
\hline TOTAL & 7 & $3.3 \%$ & 55 & $26.1 \%$ & 148 & $70.1 \%$ & 1 & $0.5 \%$ & & & \\
\hline
\end{tabular}

En el análisis bifactorial entre la variable sociodemográfica edad y la calidad de atención percibida, se halló una asociación más frecuente entre la calidad de atención de nivel muy bueno con el grupo etario de 20-25 años (en 48 encuestadas, 22.7\%). La prueba estadística encontró asociación significativa entre ambas variables ( $p$-valor > 0.05 ) con un X2 de 25.31 y $p=0.013$.
Tabla5. Grado de Instrucción y calidad de atención del parto en puérperas del Servicio de Obstetricia del Hospital Regional de Ica, Mayo - Setiembre 2019

\begin{tabular}{|c|c|c|c|c|c|c|c|c|c|c|c|}
\hline \multicolumn{12}{|c|}{ Calidad de atención } \\
\hline \multirow[t]{2}{*}{$\begin{array}{l}\text { Grado de } \\
\text { instrucción }\end{array}$} & \multicolumn{2}{|c|}{ Malo } & \multicolumn{2}{|c|}{ Bueno } & \multicolumn{2}{|c|}{ Muy bueno } & \multicolumn{2}{|c|}{$\begin{array}{c}\text { Extremad. } \\
\text { bueno }\end{array}$} & \multicolumn{3}{|c|}{ Prueba estadística } \\
\hline & $\mathbf{N}^{0}$ & $\%$ & $\mathbf{N}^{\circ}$ & $\%$ & $\mathrm{~N}^{\circ}$ & $\%$ & $\mathbf{N}^{\circ}$ & $\%$ & $x_{2}$ & gl & p \\
\hline Primaria inc & 0 & 0.0 & 0 & 0.0 & 1 & 0.5 & 0 & 0.0 & & & \\
\hline Primaria com & 0 & 0.0 & 6 & 2.8 & 0 & 0.0 & 1 & 0.5 & & & \\
\hline Secund. Incom & 0 & 0.0 & 13 & 6.2 & 36 & 17.1 & 0 & 0 & & & \\
\hline Secund. Comp. & 7 & 3.3 & 26 & 12.3 & 59 & 28.0 & 0 & 0.0 & 61,54 & 15 & 0.000 \\
\hline Superior No Univ. & 0 & 0.0 & 10 & 4.7 & 33 & 15.6 & 0 & 0.0 & & & \\
\hline Superior Univ. & 0 & 0.0 & 0 & 0.0 & 19 & 9.0 & 0 & 0.0 & & & \\
\hline TOTAL & 7 & $3.3 \%$ & 55 & $26.1 \%$ & 148 & $70.1 \%$ & 1 & $0.5 \%$ & & & \\
\hline
\end{tabular}

En el análisis bifactorial entre la variable sociodemográfica grado de instrucción y la calidad de atención percibida, se halló una asociación más frecuente entre el nivel muy bueno de calidad con la educación secundaria completa (en 59 casos, 28\%). A su vez se observa mayor asociación de nivel bueno de calidad con la instrucción secundaria completa (12.3\%). La prueba estadística encontró asociación significativa entre ambas variables ( $p$-valor > 0.05) con un X2 de 61.54 y $p=0.000$.

Tabla6. Estado civil y calidad de atención del parto en puérperas del Servicio de Obstetricia del Hospital Regional de Ica, Mayo - Setiembre 2019

\begin{tabular}{|c|c|c|c|c|c|c|c|c|c|c|c|}
\hline \multirow{3}{*}{ Estado Civil } & \multicolumn{8}{|c|}{ Calidad de atención } & & & \\
\hline & \multicolumn{2}{|c|}{ Malo } & \multicolumn{2}{|c|}{ Bueno } & \multicolumn{2}{|c|}{ Muy bueno } & \multicolumn{2}{|c|}{$\begin{array}{l}\text { Extremad. } \\
\text { bueno }\end{array}$} & \multicolumn{3}{|c|}{ Prueba estadistica } \\
\hline & $\mathbf{N}^{\circ}$ & $\%$ & $\mathbf{N}^{\circ}$ & $\%$ & $\mathbf{N}^{\circ}$ & $\%$ & $\mathbf{N}^{\circ}$ & $\%$ & $x_{2}$ & gl & p \\
\hline Soltera & 0 & 0.0 & 6 & 2.8 & 23 & 10.9 & 1 & 0.5 & \multirow{4}{*}{14.58} & \multirow{4}{*}{6} & \multirow{4}{*}{0.024} \\
\hline Casada & 0 & 0.0 & 13 & 6.2 & 16 & 7.6 & 0 & 0.0 & & & \\
\hline Conviviente & 7 & 3.3 & 36 & 17.1 & 109 & 51.7 & 0 & 0.0 & & & \\
\hline TOTAL & 7 & $3.3 \%$ & 55 & $26.1 \%$ & 148 & $70.1 \%$ & 1 & $0.5 \%$ & & & \\
\hline
\end{tabular}

En el análisis bifactorial entre la variable sociodemográfica estado civil y la calidad de atención percibida, se halló una asociación más frecuente del estado civil conviviente con el nivel muy bueno de calidad de atención del parto (en 109 casos, 51.7\%). La prueba estadística encontró asociación significativa entre ambas variables ( $p$-valor > $0.05)$ con un $\mathrm{X} 2$ de 14.58 y $p=0.024$.

Tabla7. Ocupación y calidad de atención del parto en puérperas del Servicio de Obstetricia del Hospital Regional de Ica, Mayo - Setiembre 2019

\begin{tabular}{|c|c|c|c|c|c|c|c|c|c|c|c|}
\hline \multirow{3}{*}{ Ocupación } & \multicolumn{8}{|c|}{ Calidad de atención } & & & \\
\hline & \multicolumn{2}{|c|}{ Malo } & \multicolumn{2}{|c|}{ Bueno } & \multicolumn{2}{|c|}{ Muy bueno } & \multicolumn{2}{|c|}{$\begin{array}{c}\text { Extremad. } \\
\text { bueno }\end{array}$} & \multicolumn{3}{|c|}{ Prueba estadística } \\
\hline & $\mathbf{N}^{\circ}$ & $\%$ & $\mathbf{N}^{\circ}$ & $\%$ & $\mathbf{N}^{\circ}$ & $\%$ & $\mathbf{N}^{\circ}$ & $\%$ & X2 & gl & p \\
\hline Ama de casa & 6 & 2.8 & 46 & 21.8 & 128 & 60.7 & 1 & 0.5 & \multirow{4}{*}{8.60} & \multirow{4}{*}{6} & \multirow{4}{*}{0.197} \\
\hline Independiente & 1 & 0.5 & 0 & 0.0 & 3 & 1.4 & 0 & 0.0 & & & \\
\hline Dependiente & 0 & 0.0 & 9 & 4.3 & 17 & 8.1 & 0 & 0.0 & & & \\
\hline TOTAL & 7 & $3.3 \%$ & 55 & $26.1 \%$ & 148 & $70.1 \%$ & 1 & $0.5 \%$ & & & \\
\hline
\end{tabular}

En el análisis bifactorial entre la variable sociodemográfica ocupación y la calidad de atención percibida, no se encontró una asociación estadística significativa, al obtener un X2 de 8.06 y una significancia $p=0.197$, sin proporcionales relevantes. Se evidencia que tanto la ocupación ama de casa como el ser dependiente, tienen 
en mayor proporción nivel muy bueno de calidad de atención del parto. De igual forma tanto la ocupación ama de casa como el ser dependiente tienen mayores proporciones de nivel bueno de calidad.

Tabla8. Procedencia y calidad de atención del parto en puérperas del Servicio de Obstetricia del Hospital Regional de Ica, Mayo - Setiembre 2019

\begin{tabular}{|c|c|c|c|c|c|c|c|c|c|c|c|}
\hline \multirow{3}{*}{ Procedencia } & \multicolumn{8}{|c|}{ Calidad de atención } & \multirow{2}{*}{\multicolumn{3}{|c|}{ Prueba estadística }} \\
\hline & \multicolumn{2}{|c|}{ Malo } & \multicolumn{2}{|c|}{ Bueno } & \multicolumn{2}{|c|}{ Muy bueno } & \multicolumn{2}{|c|}{$\begin{array}{l}\text { Extremad. } \\
\text { bueno }\end{array}$} & & & \\
\hline & $\mathbf{N}^{\circ}$ & $\%$ & $\mathbf{N}^{\circ}$ & $\%$ & $\mathbf{N}^{\circ}$ & $\%$ & $\mathbf{N}^{\circ}$ & $\%$ & $x^{2}$ & gl & p \\
\hline Urbano & 5 & 2.4 & 41 & 19.4 & 123 & 58.3 & 1 & 0.5 & & & \\
\hline Rural & 2 & 0.9 & 14 & 6.6 & 25 & 11.8 & 0 & 0.0 & 2.49 & 3 & 0.475 \\
\hline TOTAL & 7 & $3.3 \%$ & 55 & $26.1 \%$ & 148 & $70.1 \%$ & 1 & $0.5 \%$ & & & \\
\hline
\end{tabular}

En el análisis bifactorial entre la variable sociodemográfica procedencia y la calidad de atención percibida, no se encontró una asociación estadística significativa entre ambas variables, al obtener un X2 de 2.49 y una significancia $p=0.475$, sin diferencias proporcionales relevantes. Se evidencia que tanto la procedencia urbana como la rural, tienen la mayor proporción de nivel muy bueno de calidad (58.3\% y $11.8 \%$ respectivamente). Asimismo existe mayor proporción de nivel bueno de calidad tanto en la procedencia urbana como en la rural (19.4\% y $6.6 \%$ respectivamente).

Tabla9. Personal de salud que atendió el parto y calidad de atención en puérperas del Servicio de Obstetricia del Hospital Regional de Ica, Mayo - Setiembre 2019

\begin{tabular}{|c|c|c|c|c|c|c|c|c|c|c|c|}
\hline \multirow{3}{*}{$\begin{array}{l}\text { Personal de } \\
\text { salud }\end{array}$} & \multicolumn{8}{|c|}{ Calidad de atención } & \multirow{2}{*}{\multicolumn{3}{|c|}{ Prueba estadística }} \\
\hline & \multicolumn{2}{|c|}{ Malo } & \multicolumn{2}{|c|}{ Bueno } & \multicolumn{2}{|c|}{ Muy bueno } & \multicolumn{2}{|c|}{ Extremad. } & & & \\
\hline & $\mathbf{N}^{\circ}$ & $\%$ & $\mathbf{N}^{\circ}$ & $\%$ & $\mathbf{N}^{\circ}$ & $\%$ & $\mathbf{N}^{\circ}$ & $\%$ & \multicolumn{3}{|c|}{$\begin{array}{lll} & \\
2 & \mathrm{gl} & \mathrm{p}\end{array}$} \\
\hline Médico & 0 & 0.0 & 4 & 1.9 & 13 & 6.2 & 0 & 0.0 & \multirow{4}{*}{2.78} & \multirow{4}{*}{6} & \multirow{4}{*}{0.836} \\
\hline Obstetra & 3 & 1.4 & 30 & 14.2 & 84 & 39.8 & 1 & 0.5 & & & \\
\hline Interno de Med. & 4 & 1.9 & 21 & 10.0 & 51 & 24.2 & 0 & 0.0 & & & \\
\hline TOTAL & 7 & $3.3 \%$ & 55 & $26.1 \%$ & 148 & $70.1 \%$ & 1 & $0.5 \%$ & & & \\
\hline
\end{tabular}

En el análisis bifactorial entre la variable atención del parto y la calidad de atención percibida, no se encontró una asociación estadística significativa entre ambas variables, al obtener un $\mathrm{X} 2$ de 2.78 y una significancia $p=0.836$, sin diferencias proporcionales relevantes. Se evidencia que tanto el profesional obstetra como el interno de medicina y médico obtuvieron mayor proporción de nivel muy bueno de calidad de atención. Asimismo, se evidencia que el nivel bueno de calidad es mayormente característico en el obstetra, interno de medicina y médico $(14,2 \%, 10 \%$, y $1.9 \%$ respectivamente).

\section{DISCUSIÓN}

La calidad de atención, constituye en la actualidad un tema relevante que requiere ser abordado por el sistema de salud, ya que la percepción de satisfacción por parte del usuario es un indicador fundamental para el mejoramiento del estado de salud y pronta recuperación del paciente atendido.

Al analizar las características sociodemográficas de las puérperas y de atención del parto, se encontró en mayor proporción que la edad predominante es de 20 a 25 años (31.3\%), instrucción educativa secundaria completa (43.6\%), estado civil convivientes (72\%), ocupación ama de casa (85.8\%), y procedencia zona urbana (80.6\%). La atención del parto fue mayormente realizada por el obstetra (55.9\%). Resultados que se asemejan al estudio de Ramírez quien evidenció en su estudio que en las puérperas encuestadas predomina la edad promedio de 25.7 años, el estado civil conviviente (96.2\%), y la ocupación ama de casa (96.2\%) (14). Acosta encontró en sus resultados que la edad promedio se sitúa entre los 25 años, y que la ocupación mayoritariamente eran amas de casa con $68.5 \%$ (11). El estudio de Ayala encontró en sus resultados que el estado civil predominante era el conviviente con $75.7 \%$ y la instrucción educativa fue la secundaria completa con $59.1 \%$ (13).

Según los datos estadísticos obtenidos de la aplicación de la encuesta SERVPERF, el presente estudio evidencia que la calidad de atención del parto en forma global según la satisfacción de las puérperas inmediatas es de nivel muy bueno con $70.1 \%$, con un promedio aritmético final de la calidad de atención de $X \bigotimes=5.16$ (Nivel Muy bueno), es decir que las puérperas tiene una satisfacción moderada, resultados que concuerdan con el estudio de Sánchez quien encontró satisfacción en la atención en un $75.1 \%$ (1). De igual forma el estudio de Ayala evidenció en su estudio una satisfacción del $93.3 \%$ de nivel muy bueno en las puérperas inmediatas (13). El estudio de Alvarado y Garay también encontró resultados positivos al evidenciar que cerca del $86.3 \%$ de las puérperas están satisfechas (nivel bueno y muy bueno de calidad) con la atención del parto (17), y Ramírez encontró mayor proporción de nivel muy bueno de calidad de atención del parto en puérperas, con un $76.6 \%$ (14). Sin embargo, el estudio de Vela difiere con los nuestros resultados, al evidenciar que en mayor proporción la calidad de atención del parto es regular o media, es decir con poca satisfacción en un $50 \%$ (15).

La calidad de atención del parto también se analizó mediante dimensiones, evidenciándose en cada una de ellas que las puérperas tienen en mayor proporción satisfacción con la respuesta rápida, la empatía, la seguridad, la confianza y los elementos tangibles, calificando finalmente a cada uno de ellos dentro del nivel Muy bueno de calidad. Los resultados de la calidad de atención del parto por dimensiones, concuerdan con el estudio de Ramírez, y Alvarado y Garay $(14,17)$

A valorar el promedio aritmético de cada una de las dimensiones, se evidenció que entre todas, las que mejor fueron valoradas son la empatía con $X \otimes=5.32$, confianza $X$ $\nabla=5.18$ y elementos tangibles $X \nabla=5.43$, y las menos valoradas fueron la respuesta rápida con $X \otimes=5.05$ y seguridad $X \otimes=5.04$. Si bien es cierto que todas las dimensiones han sido valoradas dentro de la satisfacción y con un nivel muy bueno de calidad de atención del 
parto, existen ciertos aspectos de la atención que condicionan la existencia de un $3.3 \%$ de puérperas insatisfechas que valoraron la calidad de atención del parto en el nivel malo, evidenciándose que $11.4 \%$ tiene insatisfacción al no tener una atención inmediata al llegar al hospital, $11.9 \%$ con insatisfacción al no tener una rápida ayuda/atención del personal de salud, 8.5\% con insatisfacción al no tener un rápido traslado al centro obstétrico, $13.8 \%$ con insatisfacción al percibir indiferencia del personal para tratar su dolor, $10.4 \%$ con insatisfacción al percibir que le dejaron sola durante largo periodo de tiempo, $8 \%$ con insatisfacción porque no le pidieron permiso para realizar los procedimientos médicos, $45.5 \%$ con insatisfacción al no permitírsele estar acompañada con su pareja o familiar, $14.2 \%$ con insatisfacción al no permitírsele escoger la posición para dar a luz, $19.9 \%$ con insatisfacción al permitírsele consumir alimentos durante el trabajo de parto, y $14.2 \%$ con insatisfacción al no tener privacidad durante los exámenes realizados.

Según características sociodemográficas de las puérperas y personal que atendió el parto, se realizó un análisis bifactorial, encontrándose una asociación estadística significativa con la calidad de atención en las variables edad ( $p$ 0.013), grado de instrucción ( $p$ 0.000), y estado civil ( $p$ 0.024), observándose asociación más frecuente entre el grupo etario de 20-25 años con la calidad de atención de nivel muy bueno con (22.7\%). El grado de instrucción secundaria se asoció mayormente con el nivel muy bueno de calidad, y el estado civil conviviente tuvo una mayor asociación con la calidad de atención de nivel muy bueno (51.7\% del total). Las variables que no se asociaron a la calidad de atención del parto fueron la ocupación ( $p$ 0.197), procedencia ( $p$ 0.475), y personal que atendió el parto ( $p$ 0.836).

En conclusión la calidad de atención del parto, según satisfacción de las puérperas inmediatas del Servicio de Obstetricia del Hospital Regional de Ica, es en promedio de nivel muy bueno en forma global como por dimensiones; siendo las dimensiones mejor valoradas: los elementos tangibles, la empatía y la confianza, y las menos valoradas la respuesta rápida y la seguridad. Asimismo se encontró asociación estadística significativa entre las variables sociodemográficas edad, grado de instrucción, y estado civil. Las variables no asociadas fueron la ocupación, procedencia, y personal que atendió el parto. 


\section{REFERENCIAS BIBLIOGRÁFICAS}

1. Sánchez SM. Perspectiva socio sanitaria en torno a la Atención al parto en Nikki, Benín (África) [Tesis de Pregrado]. España: Universidad de Valladolid; 2017. Disponible en:

http://uvadoc.uva.es/handle/10324/24429

2. Gerónimo $C R$, Magaña $C M$, Rivas AV, Sánchez LL, Cruz LA, Morales RF. Satisfacción con la calidad de la atención en mujeres en periodo posparto de un hospital de tercer nivel, Tabasco, México. CONA MED. 2016; 21 (1): 6. Disponible en:

https://www.medigraphic.com/pdfs/conamed/con-201 6/con161c.pdf

3. Boladeras CM. La atención sanitaria al parto $y$ nacimiento: propuestas y cambio. En: Goberna TC (coords.). España: edición de la Universidad de Barcelona; 2016. Pág 115-136. Disponible en: https://books.google.com.pe/books/about/Bio\%C3\%A 9tica_de_la_maternidad_Humanizaci\%C3\%B3n.html?id $=\mathrm{V} 3 \mathrm{q} 0 \mathrm{DgAAQBAJ} \&$ printsec $=$ frontcover\&source $=$ kp_r ead_button\&redir_esc $=\mathrm{y} \# \mathrm{v}=$ onepage $\& \mathrm{q} \& \mathrm{f}=$ false

4. García RY, Anaya GJ, Acosta LM, Álvarez MM, López AE, Vásquez FT. Satisfacción de las mujeres atendidas con el parto culturalmente adecuado en Loreto, Orellana (2016), Ecuador. Rev Cubana Obstet Ginecol. 2016; 42 (4): 8. Disponible en:

http://scielo.sld.cu/scielo.php?script=sci_arttext\&pid= S0138-600X2016000400006

5. Valdez SR, Salazar AY, Rojas CA, Arenas ML. El abuso contra las mujeres durante la atención del parto en hospitales públicos en México. CONA MED. 2016; 21 (1): 6. Disponible en:

https://www.medigraphic.com/pdfs/conamed/con-201 6/cons161e.pdf

6. Recio AA. La atención al parto en España: Cifras para reflexionar sobre un problema, España. DILEMATA. 2015; 7 (18): 13. Disponible en:

https://dialnet.unirioja.es/servlet/articulo?codigo $=510$ 6931

7. Uribe TC, Contreras MA, Bravo VP, Villarroel PL, Abarzúa CF. Modelo de asistencia integral del parto: Concepto de integralidad basado en la calidad y seguridad, Santiago, Chile. REV CHIL OBSTET GINECOL. 2018; 83(3): 11. Disponible en:

https://scielo.conicyt.cl/scielo.php?script=sci_arttext\& pid $=$ S0717-75262018000300266

8. Rodríguez CS, De La Cruz VJ, Roldán AL, Terukina TR, Placencia MM, Moncada CR. "Nivel de satisfacción de gestantes Atendidas según tipo de parto en la clínica Good Hope, Febrero - Abril 2016", Lima, Perú. Rev. Fac. Med. Hum. 2017; 17(1):7. Disponible en:

http://cybertesis.unmsm.edu.pe/handle/cybertesis/756 4

9. Peñalva HO. Satisfacción de las usuarias con la atención del parto horizontal en el Hospital Carlos Monge Medrano y parto vertical en el Centro de Salud Santa Adriana Juliaca 2015 [Tesis de postgrado]. Perú: Escuela de Post Grado, Universidad andina "Néstor Cáceres Velásquez"; 2015. Disponible en:
http://revistas.urp.edu.pe/index.php/RFMH/article/vi ew/744

10. Boissy A, Windover A, Bokar A, Karafa M, Neuendorf K, Frankel R, Merlino J, Rothberg M. Communication Skills Training for Physicians Improves Patient Satisfaction, EEUU. J Gen Intern Med 31(7): 7. Disponible en:

https://www.ncbi.nlm.nih.gov/pubmed/26921153

11. Acosta N. Satisfacción materna con la atención hospitalaria proporcionada en una Institución Publica [Tesis de Postgrado]. México: Universidad autónoma San Luis de Potosí; 2015. Disponible en: https://ninive.uaslp.mx/xmlui/bitstream/handle/i/391 0/MAE1STM01501.pdf? sequence=3\&isAllowed $=y$

12. Conesa FB, Canteras JM, Ballesteros MC, Carrillo GC, Martínez RM. Comparative study analyzing women's childbirth satisfaction and obstetric outcomes across two different models of maternity care, España. BMJ OPEN. 2016; 6 (1): 10. Disponible en: https://bmjopen.bmj.com/content/6/8/e011362

13. Ayala GG. Calidad de la atención del parto según percepción de las usuarias en el servicio de centro obstétrico en el Instituto Nacional Materno Perinatal. Enero-Febrero 2015 [Tesis de pregrado]. Perú: Universidad Nacional Mayor de San Marcos; 2015. Disponible en:

http://cybertesis.unmsm.edu.pe/bitstream/handle/cy bertesis/4059/Ayala_gg.pdf? sequence $=1$

14. Ramírez ST. Percepción de la calidad de Atención del parto según las puérperas en el centro de salud San Nicolás -Ancash, 2015 [Tesis de pregrado]. Perú: Universidad Privada Arzobispo Loayza; 2015. Disponible en: http://repositorio.ual.edu.pe/handle/UAL/58

15. Vela CG. "Percepción de la paciente sobre la atención del parto humanizado en el Servicio de Centro Obstétrico del Instituto Nacional Materno Perinatal durante el periodo marzo-abril de 2015" [Tesis de pregrado]. Perú: Universidad Nacional Mayor de San Marcos; 2015.2 Disponible en: http://cybertesis.unmsm.edu.pe/bitstream/handle/cy bertesis/4278/Vela_cg.pdf?sequence $=1$

16. Ñahuis AG. Percepción de la gestante sobre la atención del parto humanizado en SANNA clínica san Borja-enero a marzo, 2017 [Tesis de postgrado]. Perú: Escuela de Postgrado, Universidad Cesar Vallejo; 2017. Disponible en:

http://repositorio.ucv.edu.pe/bitstream/handle/UCV/ 24148/\%C3\%91ahuis_AG.pdf?sequence=1\&isAllowe $\mathrm{d}=\mathrm{y}$

17. Álvaro LC, Garay HA. Percepción de las puérperas sobre la calidad de Atención del parto vaginal en el Hospital Sagaro ii-1 de tumbes. 2016 [Tesis de pregrado]. Perú: Universidad Nacional de Tumbes; 2016. Disponible en:

http://repositorio.untumbes.edu.pe/handle/UNITUM BES/75 
18. Rodríguez JM, Maque MC. Factores relacionados al nivel de satisfacción de las usuarias en la atención de parto eutócico en el Centro Obstétrico del Hospital Regional Honorio Delgado Espinoza, Arequipa Diciembre 2016-Enero 2017 [Tesis de pregrado]. Perú: Universidad Católica de Santa María; 2017. Disponible en:

http://tesis.ucsm.edu.pe/repositorio/handle/UCSM/65
14

19. Munares GO. Características biosociales, reproductivas y obstétricas asociadas al resultado adecuado del parto en Ica, Perú, 2013. MED UIS. 2015; 28 (3): 9. Disponible en:

http://www.scielo.org.co/scielo.php?script=sci_abstra ct\&pid=S0121-03192015000300004

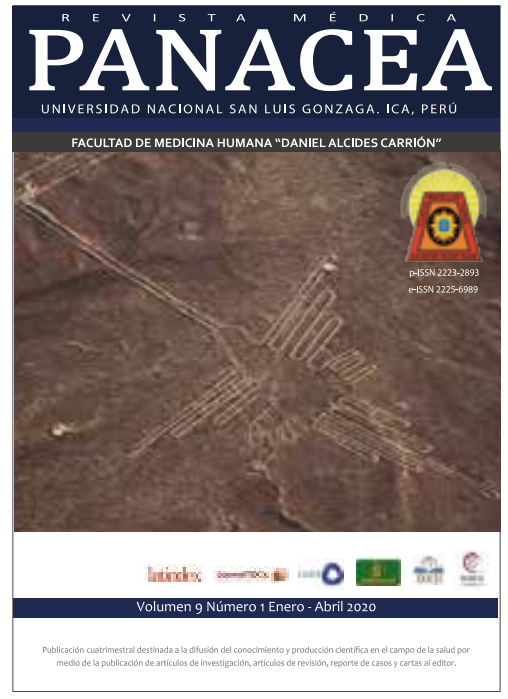

